\title{
APPLYING SDBC IN THE CULTURAL-HERITAGE SECTOR
}

\author{
Boris Shishkov \\ Department of Computer Science, University of Twente, 5 Drienerlolaan, Enschede, The Netherlands \\ Email: b.b.shishkov@ewi.utwente.nl \\ Jan L.G. Dietz \\ Department of Software Technology, Delft University of Technology, 4 Mekelweg, Delft, The Netherlands \\ Email: j.l.g.dietz@ewi.tudelft.nl
}

Keywords: $\quad$ SDBC; Software broker; Cultural heritage

Abstract: An actual cultural-heritage-related problem is how to effectively manage the global distribution of digitized cultural and scientific information, taking into account that such a global distribution is only doable through the Internet. Hence, adequately designing software applications realizing brokerage functionality in the global space, particularly concerning digitized cultural/scientific information, is to be considered as an essential cultural-heritage-related task. However, due to its great complexity, the usage of the existing popular modelling instrumentarium seems insufficiently useful; this is mainly because the realization of a satisfactory cultural-heritage brokering requires a deep understanding and consideration of the original business reality. Inspired by this challenge, we have aimed at exploring relevant strengths of the SDBC approach which is currently being developed. SDBC's being capable of properly aligning business process modelling and software specification, allowing for re-use and being consistent with the latest software design standards, are among the facts in support of the claim that SDBC could bring value concerning the design of cultural-heritage-related brokerage applications. Hence, in this paper we motivate and illustrate the usefulness of SDBC for the cultural-heritage sector.

\section{INTRODUCTION}

The latest ICT (Information and Communication Technology) developments could bring numerous societal benefits, among which is the possibility for a global access to cultural/scientific manuscripts located at any place. The actuality of this issue could be seen from a number of (currently progressing) culture-heritage-related projects, such as the project DigiCult (DigiCult, 2004). According to such projects (to most of them), several types of activities are observed, concerning the digitization of cultural and scientific heritage, among which are:

- the classification of existing cultural heritage materials;

- the recognition and processing of images from such materials;

- the specification and maintenance of the metadata related to digitized materials;

- the management and distribution of the digitized cultural/scientific materials.

In the current paper, we report further studies related to the last of the above mentioned issues. A previous study (Shishkov, 2004) has started the exploration of the strengths of SDBC concerning this particular cultural-heritage-related problem.

As stated in the above-mentioned study, in tune with the current technological possibilities and user demands, such management and distribution (of digitized cultural materials) is to be considered in global respect. It is essential that the digitized cultural/scientific materials are globally available to the public. Next to that, their accessibility must be adequately regulated, which adds complexity: some materials are to be accessible freely by anyone, others should be accessible only by authorized users, still others are to be distributed commercially, and so on. Thus, an advanced brokerage functionality needs to be realized in this regard. As far as the 'global information space' is concerned, this job is to be done by a software broker. By 'software broker' it is meant a software application which realizes a brokerage functionality. As it is well-known, software brokers are built and used for a number of purposes, for instance, flight/accommodation reservations, e-Business, Tele-Work, and so on. 
However, a global management and distribution of digitized cultural (and scientific) data, which is characterized by a number of restrictions (as mentioned above), makes the brokerage task more complex than what is usually observed in brokerage systems. It is essential, therefore, specifying such a (software) brokerage system, based on a sound consideration of the original business system, to be supported by it. This leads to a more general actual research problem, namely the alignment between business process modelling and software specification.

The SDBC (SDBC stands for Software Derived from Business Components) approach has been introduced (Shishkov \& Dietz, 2004-1; Shishkov \& Dietz, 2004-2) and considered also in another paper from the current Proceedings, as an approach being capable of adequately addressing the businesssoftware alignment by considering 'logical' components that represent the logical building blocks of a software system. In particular, the approach allows for deriving pure business process models (called business coMponents) and reflecting them in conceptual (UML-driven) software specification models (called software coMponents). In the business coMponent identification, SDBC follows a multi-aspect business perspective, guaranteeing completeness. In the business coMponent - software coMponent mapping, SDBC follows rigorous rules, guaranteeing adequate alignment. Being UML-driven, SDBC is in tune with the current software design standards.

The aim of this paper is to add further evidence in support of the claim that the (SDBC) approach could be useful with regard to the cultural-heritage sector. The paper uses and further considers the example presented in (Shishkov, 2004).

The paper's outline is as follows: Section 2 considers relevant cultural-heritage information. Section 3 illustrates the application of SDBC, using a small example. Section 4 contains the conclusions.

\section{THE CULTURAL HERITAGE SECTOR}

Among the institutions (in most countries) which are mainly concerned with the cultural heritage issue are the archival, library, and museum institutions. Such is the case in The Netherlands, for instance, where these institutions take part in the specification of the Dutch national long-term cultural strategy, addressing the cultural-heritage-related issues (it is called in The Netherlands, Cultuurnota (http://www.cultuurnota.nl)). The situation in other countries (such as Bulgaria, for example) is similar.
In the majority of the national cultural strategies the actuality of the cultural heritage issue is recognized, and especially the need to allow the cultural heritage sector adequately benefit from the current technical and technological possibilities. That is why more and more (EU) projects appear, addressing culturalheritage-related problems. An example of such a project is the DigiCult project (DigiCult, 2004). It is claimed (not only within this mentioned project) that the mere existence of technical and technological possibilities does not mean that they are straightforwardly utilizable, particularly in such a specific domain. What is required is that a clear perception of the original business (cultural heritage) is reflected in the technical/technological consideration. Otherwise, the (technical) support realized would only partially reflect the original requirements and its effect would be much limited. There are many examples for such partially successful cultural-heritage-related initiatives, such as the project American Memory (http://memory.loc.gov); it has delivered a digital collection of cultural materials. A gateway has been built to rich primary source materials relating to the history and culture of the United States of America. Through the web site of the project, one could access more than 7 million digital items from more than 100 historical collections. However, the project has not considered at all how the realized system could handle complex situations, such as dealing with different access levels. The project has not considered as well how such kind of system could be built for other analogous purposes and how it could operate in the context of a global cultural-heritagebrokering environment.

It is agreed in the cultural heritage community that a way to bring improvements in this direction is to succeed in designing (cultural-heritage-related) software systems which are soundly rooted in an adequate model of the original business reality.

Thus, taking into account that:

1. most of the current popular software design methods are insufficiently capable of adequately aligning business process modelling and software specification; and

2. there is an approach proposed, namely the SDBC approach, which is reflecting this particular problem, we have been inspired to explore some strengths of SDBC, relevant to the cultural heritage sector.

In the following section, we will present and partially illustrate our view on applying SDBC for solving some cultural-heritage-related problems. 


\section{APPLYING SDBC}

Offering useful advantages concerning the specification of software systems intended to support complex business systems in different domains, the SDBC approach has been applied successfully in test cases concerning the domains of e-Business and Tele-Work. It has been demonstrated how (using SDBC) a software brokerage system could be specified. As it is well known, software brokers are currently of great interest because of their wide applicability resulting from their actual (brokerage) functionality. They usually facilitate (Shishkov, 2004): the match-making of globally available information; the management of digital archives; the globalization of used data networks. Through software brokers, users could have a quick and effective match-making at low costs.

Because of the relevance of software brokers to the (discussed above) cultural-heritage-related problem, SDBC is applied (as an approach which possesses strengths concerning the businesssoftware alignment in general and the specification of software brokers, in particular) in building a cultural-heritage-sector (software) broker being able to effectively handle the management and global distribution of metadata as well as of digitized cultural/scientific information. Such a broker is to be usable on a global scale through the Internet.

In this way SDBC would stimulate the global availability of cultural/scientific information.

The particular role of SDBC in building such brokers will be only partially illustrated below, because of the limited scope of this paper. Because of the same reason, just the essential issues will be mentioned. And also, no explanations will be offered concerning the modelling techniques used. Such explanations are to be found in the SDBC sources which were mentioned before.

We first consider the cultural-heritage brokerage system-to-be very generally: we could view such a system as consisting of a number of 'sellers' (distributors) of anything and a number of 'buyers'. If we consider a general broker, it should match appropriate seller and buyer information based on some criteria.

A further analysis should follow, based on this general view, considering the particular cultural heritage information (discussed already). In SDBC, the results of such an analysis are reflected in the SCI model (interested readers could read of the SCI model in the SDBC materials).

The general SCI model relevant to the current situation is depicted, just for illustrative purpose, in Figure 1 . The model is incomplete, only some basic issues are there. Among them are: units within the
General Broker (AU (Acceptance Unit): responsible for accepting and handling submissions from sellers and buyers; FU (Financial Unit): responsible for handling the fee payments done by sellers/buyers as a compensation for the work of the broker; MM (Match-Maker): responsible for performing the match-making concerning the seller and buyer information) and actors outside the General Broker (Seller: offering/distributing something, for example, digitized cultural materials; Buyer: being interested in something, for example, in particular digitized cultural materials; Expert: responsible for assisting the broker in some complex situations (in which, for example, 'human' cultural heritage experience is required); Insurer: responsible for the insurance of relevant issues, for instance, insurance against fraud).

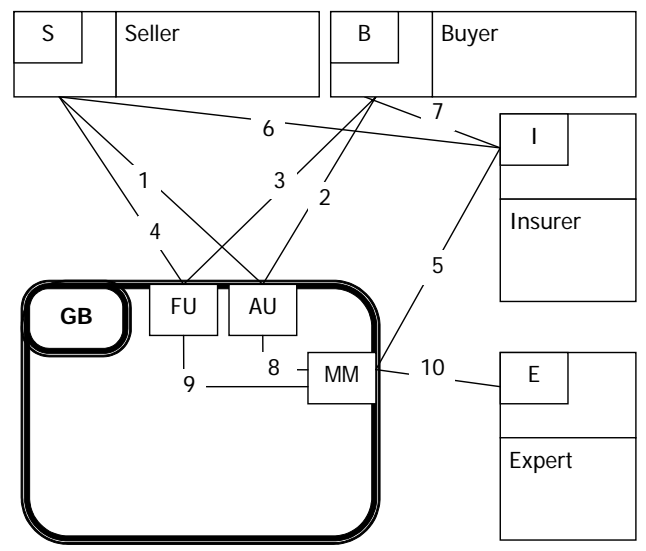

Figure 1: The General Broker (GB): SCI Model.

Hence, the GB SCI model facilitates the structuring of the initial case information to be reflected in the identification of a business coMponent.

In this particular case, it is suggested that a general business coMponent is identified. The reason relates to the wide usage of software brokers in a number of cases; thus, identifying a general model would allow for re-using it many times. Our (DEMO-based) general business coMponent is depicted in Figure 2. The model is incomplete because of its having just an illustrative purpose. More information on the DEMO modelling and the derivation of a DEMO model within SDBC could be found in the SDBC materials, mentioned before.

As seen from the Figure, two internal GB actors (actor-roles) are depicted and also two external actors ('seller' and 'buyer': they are modeled as an aggregated actor because of their having the same general attitude towards the broker). As for the internal GB actors depicted, they are the match- 
making unit ('match-maker') and the financial unit ('payment controller'). Two transactions are specified, concerning all the mentioned actors: T01 match-making (executed by the match-maker) and T02 payment (executed by seller/buyer). One transaction is specified, taking place within the GB: C3 (it concerns the periodical self-activation of the payment controller in handling all the payments related to a particular period of time). There is also a data bank depicted (EB01), containing the necessary data (concerning both buyers and sellers) that the match-maker should have in order to be able to realize a match-making.

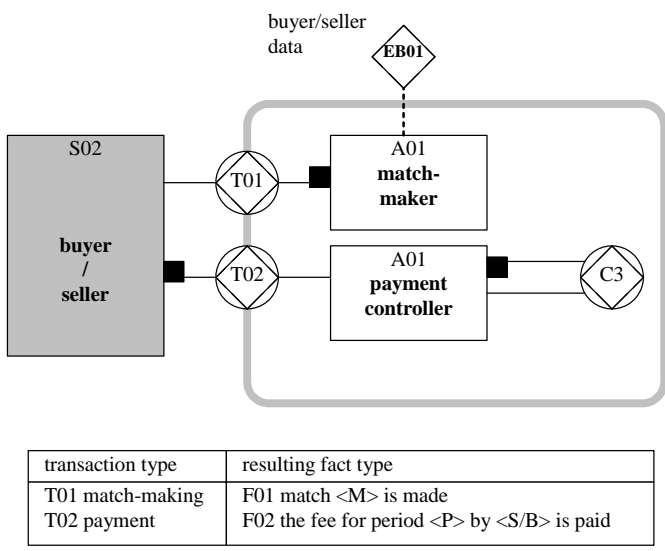

Figure 2: DEMO-based (GB) general business component.

Once built, this general business coMponent needs to be extended, according to SDBC, aiming at the identification of a particular business coMponent, in this case: Cultural heritage sector broker (built again with the DEMO notations). However, because of the limited scope of this paper, the transformation from the general business coMponent (The General Broker) to the particular business coMponent (The Cultural heritage sector Broker) is not presented. Information on how such an extension is carried out within SDBC could be found in the (mentioned) SDBC materials.

According to SDBC, a DEMO-based business coMponent (The Cultural heritage sector Broker) should be reflected in a use case software specification model. An example of such a model is depicted in Figure3. The model is incomplete, containing only some of the use cases characterizing such a broker. The broker is to use a database. It is virtually divided in two parts: one concerning the data submitted by distributors (of digitized cultural heritage materials) and the other one, concerning the data submitted by users. They are represented on the
Figure by 'DBD' and 'DBU', respectively ('DB' standing for database; $\mathrm{D}(\mathrm{U})$ standing for distributor (user)). On the Figure, it is just illustrated how a software specification model would look like. The use case model will not be explained since it is expected that most of the readers are familiar with UML. As for the DEMO - use case derivation mechanism, information on it could be found in (Shishkov \& Dietz, 2004-3).

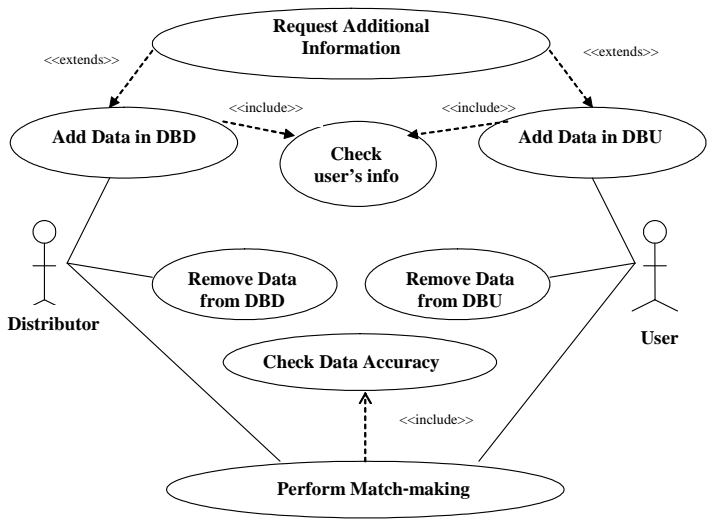

Figure 3: The Cultural-heritage-sector broker: use case software specification model.

\section{CONCLUSIONS}

This paper has provided further evidence on the practical applicability of the SDBC approach, by considering its usefulness for the cultural heritage sector. In this way, the paper complements the other SDBC-related paper within the current Proceedings.

Among the further research goals of the authors is the generalization of the results concerning the usage of SDBC for specifying brokerage systems in different domains, including Cultural heritage, eBusiness and Tele-Work. This would not only bring further evidence about the values of SDBC concerning software specification but would also result in a deliverable (a thorough general brokerage model) which is helpful for developing useful software products. To realize this, it is essential to complement the general brokerage model with rigorous rules on how to extend it depending on the particular purpose of use. The existing examples (within the mentioned domains) are to be considered as appropriately complementing such rules. The rules themselves would be defined based on the general SDBC application guidelines (Shishkov \& Dietz, 2004-1; Shishkov \& Dietz, 2004-2).

However, generalizing further, towards SDBC models which are general for any domain and concerning any functionality, would add little value 
mainly because the extension of such models would require significant efforts. Therefore, we look towards functionality/domain-specific general business coMponents through which SDBC could adequately accomplish business-software alignment and proper re-use. Otherwise said, we envision a real re-use value of applying SDBC:

- either if it grasps the core of a functionality, to be reflected in different domains;

- or if it captures essential issues for a domain, allowing for further specializations depending on the particular needs.

It is expected that these and further SDBCrelated (research) achievements would be greatly useful for the current service development which has significant societal relevance (Pires et al, 2003). SDBC could facilitate the specification of services not only by providing business-software-alignment and re-use mechanisms but also through a support towards the grasping of the context concerning the services' operation. Taking into account that such a context grasping requires a sound business modelling and its further adequate reflection in a (service) specification, it is logical to expect that SDBC (itself developed around such desired properties) might be helpful.

Therefore, the essential values of the SDBC approach, namely adequate business modelling, proper business-software alignment, and re-use, do prove to be relevant both scientifically and societally.

\section{REFERENCES}

DigiCult, 2004: http://slim.emporia.edu/globenet/sofia2004/index.htm

Pires, L. F.; M. van Sinderen, C. de Farias, J.P.A. Almeida. Use of models and modelling techniques for service development. In I3E'03, $3^{\text {rd }}$ IFIP Confeence on E-commerce, E-business, and E-government. Kluwer Academic Publishers, 2003.

Shishkov, B. Designing cultural heritage sector brokers using SDBC. In International Journal "Information Theories \& Applications” (IJ ITA), Vol. 10, 2004.

Shishkov, B. and J.L.G. Dietz, 2004-1. Aligning business process modelling and software specification in a component-based way, the advantages of SDBC. In ICEIS'04, 6th International Conference on Enterprise Information Systems. ICEIS Press, 2004.

Shishkov, B. and J.L.G. Dietz, 2004-2. Design of software applications using generic business components. In HICSS'04, 37th Hawaii International Conference on System Sciences. IEEE Computer Society Press, 2004.

Shishkov, B. and J.L.G. Dietz, 2004-3. Deriving use cases from business processes, the advantages of DEMO.
Enterprise Information Systems V, Edited by O. Camp, JB.L. Filipe, S. Hammoudi, and M. Piattini, Kluwer Academic Publishers, Dordrecht/Boston/London, 2004. 